\title{
Dielectric and Dilatometric Studies of Glass Transitions in Thin Polymer Films
}

\author{
Koji Fukao and Yoshihisa Miyamoto \\ Faculty of Integrated Human Studies, Kyoto University, Kyoto 606-8501, Japan
}

(Received November 7, 2018)

\begin{abstract}
Dielectric relaxation and thermal expansion spectroscopy were made for thin polystyrene films in order to measure the temperature $T_{\alpha}$ corresponding to the peak in the loss component of susceptibility due to the $\alpha$-process and the $\alpha$-relaxation time $\tau$ as functions of film thickness $d$. While the glass transition temperature $T_{\mathrm{g}}$ decreases with decreasing film thickness, $T_{\alpha}$ and $\tau$ were found to remain almost constant for $d>d_{\mathrm{c}}$ and decrease drastically for $d<d_{\mathrm{c}}$ for high temperatures. Here, $d_{\mathrm{c}}$ is a critical thickness. Near the glass transition temperature, the thickness dependence of $T_{\alpha}$ and $\tau$ is more prominent. The relation between the fragility index and non-exponentiallity is discussed for thin films of polystyrene.
\end{abstract}

\section{INTRODUCTION}

Understanding the behavior of the characteristic length scale of the dynamics of supercooled liquids near the glass transition is the most important problem to be solved in studies on glass transitions [1]. Glass transitions in finite systems confined to nanopores [2-4] and thin films [5-9] have recently attracted much attention, because such systems can be regarded as model systems for studying the length scale of glass transitions. In such systems, deviation from bulk properties is expected to appear if the system size is comparable to the characteristic length scale. In particular, $T_{\mathrm{g}}$ of thin films have been measured using several experimental techniques [5-8] and a drastic reduction of $T_{\mathrm{g}}$ has been observed with decreasing film thickness. However, the dynamics of the $\alpha$-process in thin polymer films has not yet been clarified in detail.

In previous papers [10], we reported that $T_{\mathrm{g}}$ for thin polystyrene films supported on glass substrate can be determined from the temperature change of the electric capacitance and that the dynamics of the $\alpha$-process can be determined from the dielectric loss of the films. We were able to obtain the distinct thickness dependences of $T_{\mathrm{g}}$ and $T_{\alpha}$ in which the dielectric loss exhibits a peak value for a fixed frequency due to the $\alpha$-process. In this paper, we investigate the dynamics of $\alpha$-process in thin polymer films, especially the thickness dependence of the dynamics for a wider frequency range, including near the glass transition temperature, by applying dielectric relaxation (DES) and thermal expansion spectroscopy (TES).

\section{EXPERIMENTALS}

Atactic polystyrene (a-PS) used in this study was purchased from the Aldrich Co., Ltd. $\quad\left(M_{\mathrm{w}}=\right.$ $1.8 \times 10^{6}, M_{\mathrm{w}} / M_{\mathrm{n}}=1.03$, where $M_{\mathrm{w}}$ and $M_{\mathrm{n}}$ are the weight average and the number average of the molecular weights, respectively). Thin films of a-PS with various thicknesses were prepared on an Al-deposited slide glass using a spin-coat method from a toluene solution of a-PS. The thickness was controlled by changing the concentration of the solution. After annealing at $70^{\circ} \mathrm{C}$ in the vacuum system for several days to remove solvents, $\mathrm{Al}$ was vacuum-deposited again to serve as an upper electrode. Heating cycles in which the temperature was changed between room temperature and $110^{\circ} \mathrm{C}\left(>T_{\mathrm{g}}\right)$ were applied prior to the dielectric measurements to relax the as-spun films and obtain reproducible results. Dielectric measurements were done using an LCR meter (HP4284A) in the range of frequency of applied electric field, $f_{\mathrm{E}}$, from $20 \mathrm{~Hz}$ to $1 \mathrm{MHz}$ during heating (cooling) processes in which the temperature was changed at a rate of $2 \mathrm{~K} / \mathrm{min}$ or $0.5 \mathrm{~K} / \mathrm{min}$.

Thermal expansion spectroscopy is a new technique which has very recently been introduced in studies on slow dynamics supercooled liquids by Bauer et al [11]. In this method, a sinusoidal temperature modulation, $T(t)=\langle T\rangle+T_{\omega} e^{i \omega t}$, is given to the sample, and then corresponding change 
in capacitance with the same angular frequency as the temperature modulation has, $C^{\prime}(t)=\left\langle C^{\prime}\right\rangle+$ $C_{\omega}^{\prime} e^{i(\omega t+\delta)}$, is detected within a linear response region. Here, $\omega=2 \pi f_{\mathrm{T}}$ and $f_{\mathrm{T}}$ is the frequency of temperature modulation. Because this capacitance change is directly connected with the volume change in thin films, the volume change can be obtained in accordance with the applied temperature modulation. Furthermore, the same sample can be used both for DES and TES without changing measurement conditions. In case of thin films of a-PS in which the area of the film surface remains constant with temperature change, the temperature coefficient $\tilde{\alpha}$ of capacitance $C^{\prime}$ and the linear thermal expansion coefficient, $\alpha_{\mathrm{n}}$, normal to the film surface satisfy the following relation: $\tilde{\alpha} \equiv$ $-\frac{1}{C^{\prime}\left(T_{0}\right)} \frac{d C^{\prime}}{d T} \approx 2 \alpha_{\mathrm{n}}$, where $C^{\prime}(T)$ is the capacitance at temperature $T$ and $T_{0}$ is a standard temperature. Using the amplitude of sinusoidal temperature modulation $T_{\omega}$ and that of the response $C_{\omega}^{\prime}, \tilde{\alpha}$ is given by the equation: $\tilde{\alpha}=\frac{1}{C^{\prime}\left(T_{0}\right)} \frac{C_{\omega}^{\prime}}{T_{\omega}}$. In the present measurements an average temperature $\langle T\rangle$ is controlled to increase with a constant rate from $0.1 \mathrm{~K} / \mathrm{min}$ to $0.5 \mathrm{~K} / \mathrm{min}$. The amplitude $T_{\omega}$ is set from $0.2 \mathrm{~K}$ to $0.6 \mathrm{~K}$. For capacitance measurement in TES the frequency of applied electric field was chosen to be $100 \mathrm{kHz}$ to avoid the interference with dielectric relaxation.

\section{DIELECTRIC RELAXATION SPECTROSCOPY}

Dielectric relaxation spectroscopy for the frequency range $f_{\mathrm{E}}=20 \mathrm{~Hz} \sim 1 \mathrm{MHz}$ reveals the dynamics of the $\alpha$-process of a-PS at temperatures higher than the glass transition temperature $\left(T_{\mathrm{g}} \sim 370 \mathrm{~K}\right)$ by ca. $20 \mathrm{~K} \sim 40 \mathrm{~K}[10]$. The results obtained in this frequency and temperature range can be summarized as follows: 1) The $d$ dependence of $T_{\mathrm{g}}$ is directly correlated to the distribution of relaxation times of the $\alpha$-process. 2) The temperature at which dielectric loss is maximal due to the $\alpha$-process in the temperature domain and the $\alpha$-relaxation time obtained by the frequency dependence of the dielectric loss remain constant down to the critical thickness $d_{\mathrm{c}}$, while below $d_{\mathrm{c}}$ they decreases drastically with decreasing thickness. These results are shown in Figs.2-4 with those of TES, where the observed points by dielectric relaxation spectroscopy are plotted with the label "DES".

\section{THERMAL EXPANSION SPECTROSCOPY}

Figure 1 shows the temperature change in both real and imaginary parts of dynamical thermal expansion coefficient $\alpha_{\mathrm{n}}$ at a frequency $f_{\mathrm{T}}=16.7 \mathrm{mHz}$ of temperature modulation. In case of $d=362 \mathrm{~nm}$ (bulk sample) the loss peak exists at around $378 \mathrm{~K}$, while in case of films with $d=18 \mathrm{~nm}$ the peak temperature is shifted to lower temperature. At the same time, the peak width clearly increases with decreasing film thickness. This $d$-dependence obtained by TES for low frequencies is quite similar to that obtained by DES for higher frequencies [10]. In bulk sample, the change in thermal expansion coefficient due to the glass transition occurs in a narrow temperature range, while in very thin films of a-PS it changes in a wider temperature range. In Fig.1, the thermal expansion coefficient of glassy state, $\alpha_{\mathrm{g}}$, is smaller than that in $d=362 \mathrm{~nm}$ at $f_{\mathrm{T}}=16.7 \mathrm{mHz}$. However, it is observed that $\alpha_{\mathrm{g}}$ depends
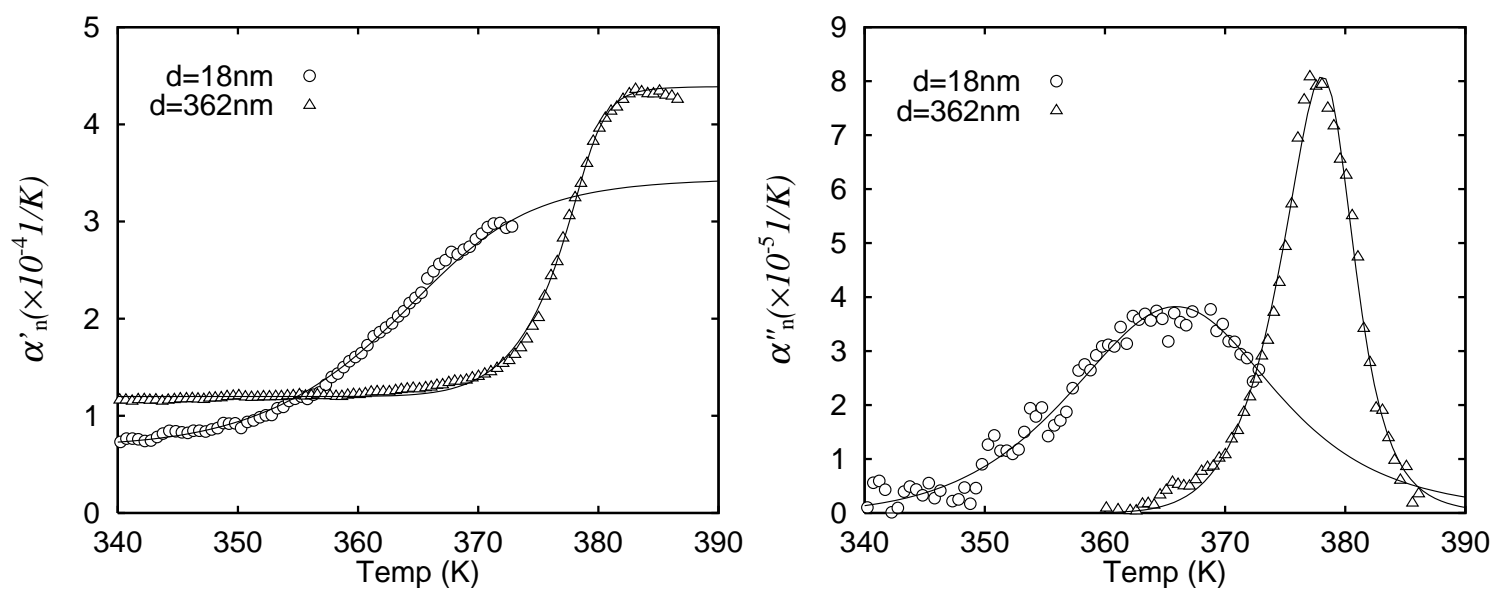

Figure 1. Temperature dependence of complex linear thermal expansion coefficient $\alpha_{\mathrm{n}}$ for a-PS with film thickness $18 \mathrm{~nm}$ and $362 \mathrm{~nm}\left(f_{\mathrm{T}}=1.67 \times 10^{-2} \mathrm{~Hz}\right)$. The left figure shows the real part of $\alpha_{\mathrm{n}}$ and the right one, the imaginary part. Solid lines are calculated by using the HN equation and the VFT equation. 


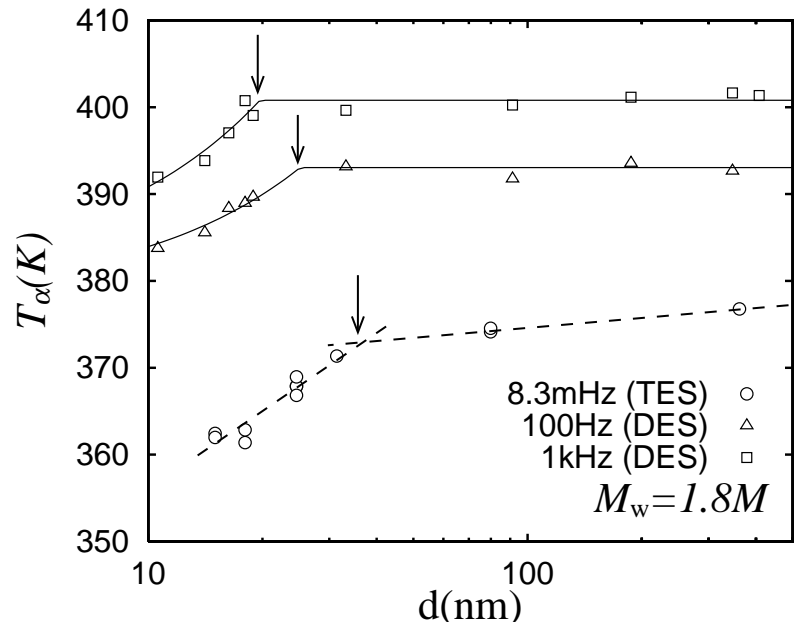

Figure 2. $d$ dependence of $T_{\alpha}$ of a-PS films obtained by DES and TES. The arrows show critical thickness at which a drastic decrease in $T_{\alpha}$ begins.

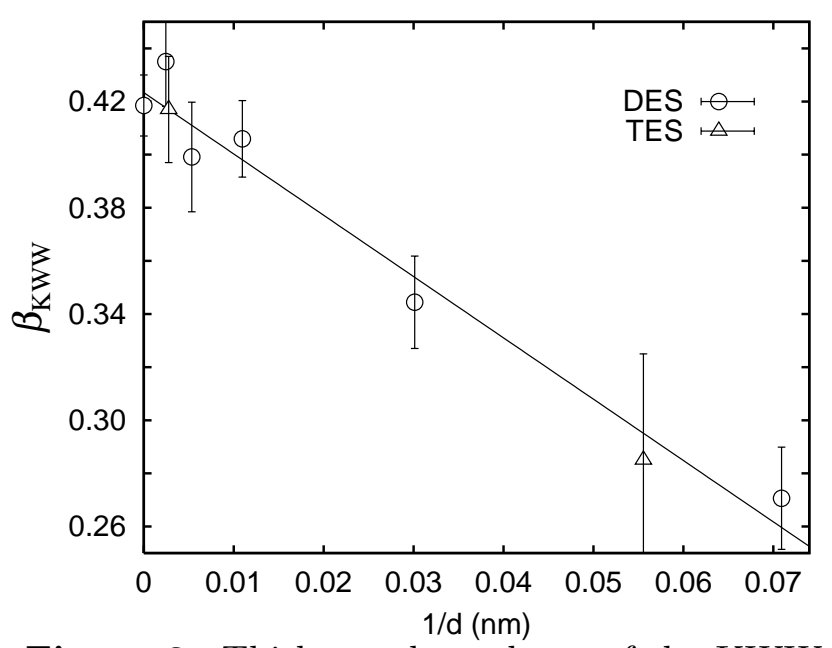

Figure 3. Thickness dependence of the KWW exponent $\beta_{\mathrm{KWw}}$ obtained by $\operatorname{DES}(\mathrm{o})$ and $\operatorname{TES}(\triangle)$.

on $f_{\mathrm{T}}$, i.e., $\alpha_{\mathrm{g}}$ increases with decreasing $f_{\mathrm{T}}$ and, as a result, $\alpha_{\mathrm{g}}$ for $d=18 \mathrm{~nm}$ is larger than that for $d=362 \mathrm{~nm}$ for the frequency $f_{\mathrm{T}}$ smaller than ca. $4 \mathrm{mHz}$, which is consistent with the results obtained in previous works [5, 10].

\section{DYNAMICS OF $\alpha$-PROCESS IN a-PS THIN FILMS}

The $d$ dependence of $T_{\alpha}$ obtained by TES is shown in Fig.2, together with that obtained by DES. The $d$ dependence of $T_{\alpha}$ observed by TES is qualitatively similar to that by DES, although no detailed $d$ dependence of $T_{\alpha}$ can so far be obtained by TES because of the limiting number of the data with different thickness. Nevertheless, it is clearly seen that the critical thickness $d_{\mathrm{c}}$ at which drastic decrease in $T_{\alpha}$ begins with decreasing film thickness shifts to a larger value of $d$ as the frequency of applied field decreases. A slight decrease can also be seen even in films with $d \approx 80 \mathrm{~nm}$ for $f_{\mathrm{T}}=8.3 \mathrm{mHz}$ by TES. Near the glass transition temperature the $d$ dependence is more prominent compared with that at higher temperatures.

The dependence of $\alpha_{\mathrm{n}}$ on temperature can be reproduced by assuming two empirical equations, as shown in Fig.1. The solid curves in Fig.1 are calculated by using the Havriliak-Negami (HN) equation $\alpha_{\mathrm{n}}(\omega)=\alpha_{\mathrm{g}}+\Delta \alpha /\left(1+(i \omega \tau)^{\alpha}\right)^{\beta}$ and the Vogel-Fulcher-Tammann (VFT) equation $\tau(T)=\tau_{0} \exp (U /(T-$ $\left.T_{V}\right)$ ), where $\Delta \alpha=\alpha_{1}-\alpha_{\mathrm{g}}, \alpha_{1}$ is the thermal expansion coefficient of the liquid state, $\alpha$ and $\beta$ are the shape parameters, $\tau_{0}$ and $U$ are constants, and $T_{\mathrm{V}}$ is the Vogel temperature. Fitting the data in Fig. 1 to the two equations, the shape parameters $\alpha$ and $\beta$ can be obtained: $\alpha=0.46$ and $\beta=0.46$ for $d=18 \mathrm{~nm}$, and $\alpha=0.85$ and $\beta=0.40$ for $d=362 \mathrm{~nm}$. The exponent $\beta_{\mathrm{KWw}}$ of the KWW relaxation function, $\phi(t)=\exp \left(-(t / \tau)^{\beta_{\mathrm{KWw}}}\right)$, can be estimated from the values of $\alpha$ and $\beta$ using the empirical relation $\beta_{\mathrm{KWw}} \approx(\alpha \beta)^{1 / 1.23}$. As shown in Fig.3, it seems that the values of $\beta_{\mathrm{Kww}}$ obtained by DES and TES fall on the same line, and that $\beta_{\mathrm{Kww}}$ becomes smaller as $d$ decreases. This result suggests that the distribution of the $\alpha$-relaxation times becomes broader as the thickness decreases. This broadening may be due to the dynamical heterogeneity within thin films which is enhanced as the temperature approaches $T_{\mathrm{g}}$.

We measured the temperature $T_{\alpha}$ for a given frequency $f_{\mathrm{E}}$ and $f_{\mathrm{T}}$, and also the frequency $f_{\max }$ at which the loss component has a maximum at a given temperature. The data observed in both measurements by using both DES and TES can be summarized as shown in Fig.4, which shows the dispersion map of the $\alpha$-process in thin a-PS films for a frequency range from $10^{-3} \mathrm{~Hz}$ to $10^{4} \mathrm{~Hz}$. The data measured for the films with the same or similar thickness are connected with each other using the VFT equation with same parameters in order to obtain a temperature dependence of $\tau$ and $T_{\alpha}$ over a wider temperature range. (See solid curves in Fig.4). As suggested in Fig.2, the $d$ dependence of the $\alpha$-relaxation time changes with frequency of applied field $\left(f_{\mathrm{T}}\right.$ and $\left.f_{\mathrm{E}}\right)$. Near the glass transition temperature, a shift of the $\alpha$-relaxation time with change in $d$ in thin films is enhanced compared with that at higher temperatures. For example, the frequency corresponding to the $\alpha$-relaxation time ranges over 3 decades at $375 \mathrm{~K}$, while it does over a half decade at $400 \mathrm{~K}$. 
If we assume that there is a characteristic length scale $\xi$ for the $\alpha$-process in this system, we can expect that the length scale $\xi$ increases as the temperature approaches to $T_{\mathrm{g}}$. Accordingly, as $d$ decreases at a given temperature near $T_{\mathrm{g}}$, a deviation of $\tau$ from the values of bulk samples should begin at a larger value of $d$ than at higher temperatures, because the change of $\tau$ and $T_{\alpha}$ with $d$ becomes appreciable in case that $\xi$ reaches the thickness $d$. This behavior is quite similar to that observed in $\alpha$-dynamics in system confined in nano pores [3].

The frequency $f$ plotted in Fig. 4 is related to the $\alpha$-relaxation time $\tau$ through the relation $2 \pi f \tau=1$. Using this relation and the definition of $T_{\mathrm{g}}$ that $\tau\left(T_{\mathrm{g}}\right)=10^{2}$ sec, the data in Fig. 4 can be replotted as shown in Fig.5, where so-called Angell plot is used. In Fig.5, the fragility index $m$, which is defined by the equation $m=\left(\frac{d \log \tau(T)}{d\left(T_{\mathrm{g}} / T\right)}\right)_{T=T_{\mathrm{g}}}$, can be estimated for a-PS films. The values of $m$ for various $d$ values are as follows: $m=86$ for $d=18 \mathrm{~nm}, m=116$ for $d=30 \mathrm{~nm}$ and $33 \mathrm{~nm}$, and $m=136$ for $d=362 \mathrm{~nm}$ and $408 \mathrm{~nm}$. Hence, it is found from the present measurements that the fragility index $m$ decreases with decreasing $d$, in other words, a-PS films become less fragile as $d$ decreases. Because the exponent $\beta_{\mathrm{KWw}}$, which is a measure for non-exponentiality, decreases with decreasing $d$, as shown in Fig.3, the exponent $\beta_{\mathrm{Kww}}$ is an increasing function of the fragility index $m$.

On the other hand, it is well-known than in bulk supercooled liquids, the fragility index $m$ is strongly correlated with the exponent $\beta_{\mathrm{Kw}}$; as $m$ increases, the exponent $\beta_{\mathrm{Kww}}$ decreases, except a few groups of materials [12]. In case of thin films of a-PS, it is found that the general correlation between $m$ and $\beta_{\mathrm{Kww}}$ does not hold.

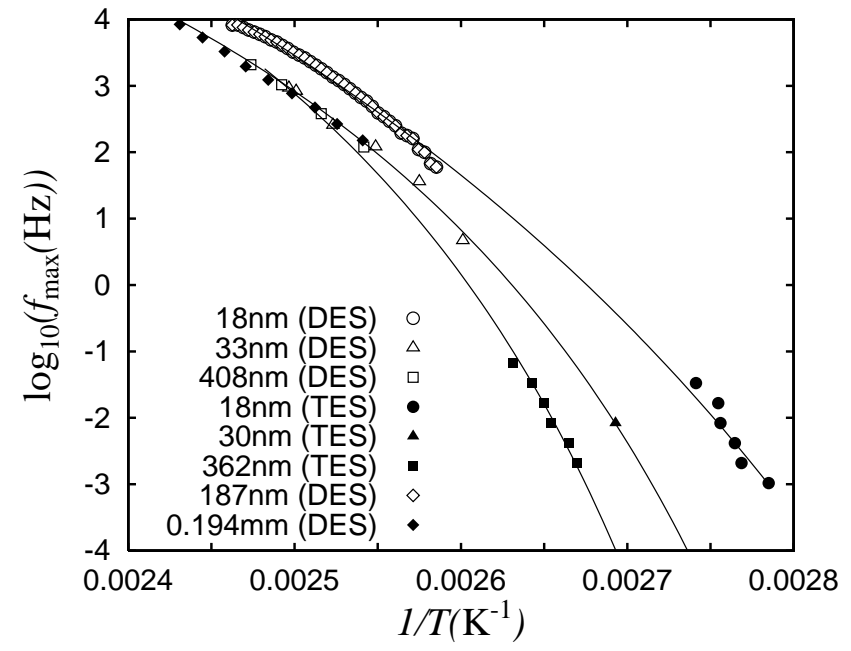

Figure 4. Dispersion map for thin films of a-PS obtained from the peak positions of the loss component $\alpha_{\mathrm{n}}^{\prime \prime}$ or $\epsilon^{\prime \prime}$ for various film thicknesses.

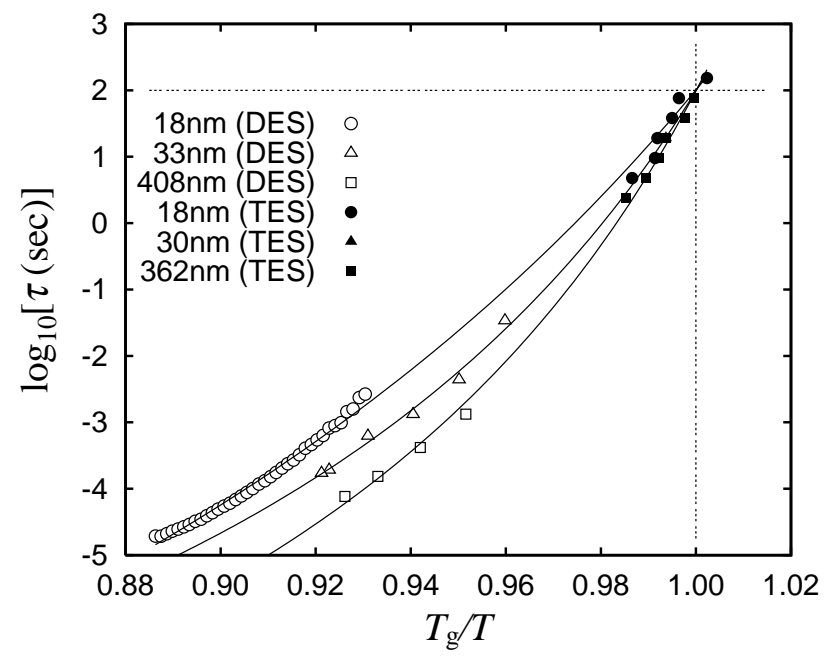

Figure 5. Relaxation time as a function of $T_{\mathrm{g}} / T$ for various values of $d$.

\section{Acknowledgments}

This work was partly supported by a Grant-in-Aid from the Ministry of Education, Science, Sports and Culture of Japan.

\section{References}

[1] M.D. Ediger, C.A. Angell, and S.R. Nagel, J. Phys. Chem. 100, 13201 (1996).

[2] J.Schüller, Yu.B. Mel'nichenko, R.Richert, and E.W.Fischer, Phys. Rev. Lett. 73, 2224(1994).

[3] M.Arndt, R.Stannarius, H.Groothues, E.Hempel, F.Kremer, Phys. Rev. Lett. 79, 2077 (1997).

[4] R. Pelster, Phys. Rev. B59, 9214 (1999).

[5] J.L. Keddie, R.A.L. Jones, and R.A. Cory, Europhys. Lett. 27, 59 (1994).

[6] G.B. DeMaggio et al., Phys. Rev. Lett. 78, 1524 (1997).

[7] J.A. Forrest, K. Dalnoki-Veress, and J.R. Dutcher, Phys. Rev. E56, 5705 (1997).

[8] W.E. Wallace, J.H. van Zanten, and W.L. Wu, Phys. Rev. E52, R3329 (1995).

[9] B. Jerome and J. Commandeur, Nature 386, 589 (1997).

[10] K. Fukao and Y. Miyamoto, Europhys. Lett. 46, 649 (1999), Phys. Rev. E, in press.

[11] C. Bauer et al., Phys. Rev. E, in press ; J. Non-Cryst. Solids, in press.

[12] R. Böhmer, K. L. Ngai, C. A. Angell, D. J. Plazek, J. Chem. Phys. 99, 4201, (1993). 